leucocytes in synovial fluid plus presence of X-ray chondrocalcinosis in at least one joint) and controls (patients with synovial effusion shown to have no CPP crystals and no chondrocalcinosis in hands and knee X-rays), paired by age and gender. Patients with hemochromatosis or primary hyperparathyroidsm were not included. General variables were included along with plausible metabolic variables ( $\mathrm{Ca}, \mathrm{P}, \mathrm{Mg}$, iPth, iron saturation [satFe\%], ferritin, diuretics and type of diuretic, and HFE genotype), and distributon of joint involvement (mono-oligopolyarticular) and clinical manifestations (acute [A-CPPCA] and chronic inflammatory [Cl-CPPCA]), as in EULAR recommendations.

Results: 340 patients and 316 controls were recruited, $53 \%$ were men, age at inclusion was $67 \pm 10$ year (IC range $62-75$ ), time from onset of symptoms 5.2 \pm 5.3 year (IC range 1-8). Regarding cases, A-CPPCA was present in 147 (43.2\%), Cl-CPPCA in 193 (56.8\%), with monoarticular involvement in 102 $(30.0 \%)$, oligoarticular in $176(51.8 \%)$, and polyarticular in $62(18.2 \%)$. Patients showed higher serum ferritine levels and lower $\mathrm{Mg}$ levels than controls (253 and 2.00 vs 204 and 2.08 , respectively), along with higher rate of HFE gene mutations (Odds ratio $2.30,95 \% \mathrm{Cl}: 1.66$ to 3.20 ). Genotypes including homozygotic mutations of H63D allele, heterozygotic mutations for $\mathrm{C} 282 \mathrm{Y}$ allele, and double heterozygotic mutations for $\mathrm{C} 282 \mathrm{Y}$ and $\mathrm{H} 63 \mathrm{D}$ were statistically associated with higher frequency of polyarticular involvement and with CI-PPA (figure 1). Clinical variables were also associated with higher SatFe\% levels, but not Mg or ferritin levels. S65C gene mutations were not increased in patients compared to controls and did not show any association with clinical phenotype.

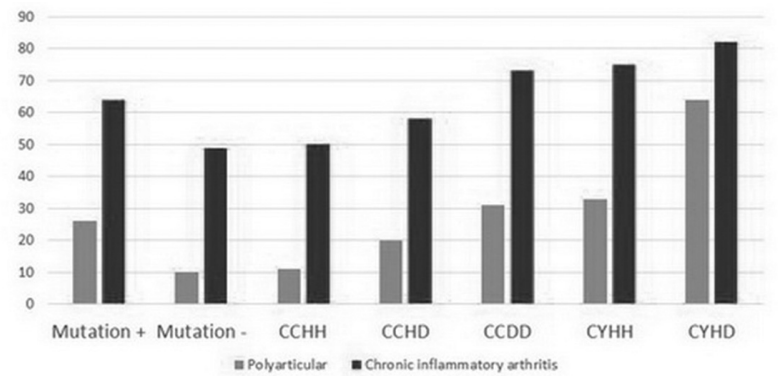

Abstract OP0183 - Table 1 Rate (\%) of polyarticular and chronic inflammatory clinical pattern impact of presence of any mutation and genotypes of FHE genes

Conclusions: Patients with definite CPPCA show differences in serum $\mathrm{Mg}$ and Ferritin levels compared to that of controls, and may contribute, along with other factors, to development of CPPCA. Nevertheless, only presence of some HFE genotypes involving $\mathrm{C} 282 \mathrm{Y}$ and $\mathrm{H} 63 \mathrm{D}$ genes were associated with more severe phenotype of clinical involvement.

Disclosure of Interest: None declared

DOI: 10.1136/annrheumdis-2018-eular.4775

\section{OP0184 THE FREQUENCY OF FLARES IN SUBJECTS WITH CHRONIC REFRACTORY GOUT TREATED WITH PEGLOTICASE IS RELATED TO VARIATION IN THE LEVEL OF PLASMA URATE}

L. Calabrese ${ }^{1}$, T. Fields ${ }^{2}$, A. Yeo ${ }^{3}$, P. Lipsky ${ }^{4} .{ }^{1}$ Cleveland Clinic, Cleveland; ${ }^{2}$ Weill Cornell Medical College, New York, ${ }^{3}$ Horizon Pharma, Lake Forest, ${ }^{4}$ AMPEL BioSolutions LLC, Charlottesville, USA

Background: Lowering plasma urate in subjects with gout is associated with an increase in flares because of an altered equilibrium between soluble urate and uric acid crystals. ${ }^{1}$ Details of the relationship between plasma urate lowering and gout flares have not been fully delineated. Pegloticase is a mammalian recombinant uricase conjugated to polyethylene glycol approved for the treatment of chronic gout refractory to conventional oral urate lowering therapy. ${ }^{2-4}$ Results from the two 6 month randomised clinical trials (RCTs) of pegloticase showed that during months $1-3$, there was an increase in pegloticase-treated subjects experiencing gout flares vs those receiving placebo. However, the reported analysis did not separate results for subjects who did and did not respond to pegloticase with a sustained reduction in plasma urate levels or relate flares to plasma urate levels. Objectives: To determine factors associated with the occurrence of flares in subjects who did and did not respond to pegloticase in the two RCTs for this therapy. Methods: This analysis used results from 2 pivotal RCTs ${ }^{2}$ of pegloticase $(8 \mathrm{mg}$ every 2 weeks [q2] or every 4 weeks [q4]) vs placebo in subjects with chronic refractory gout. A responder was defined as a subject with plasma urate $<6.0 \mathrm{mg} /$ $\mathrm{dL} \geq 80 \%$ of the time from the week-9 infusion to just prior to the week-13 infusion and from the week-21 infusion to the week-25 final study visit. Gout flares were defined as acute joint pain and swelling requiring treatment. Gout flare prophylaxis (colchicine, $0.6 \mathrm{mg}$ once or twice daily, or a NSAID) was initiated 1 week before the first infusion and continued throughout the study.

Results: There were increases in the frequency of subjects experiencing flares and the mean flare frequency in the pegloticase group vs placebo during the first 3 months of the trial (figure $1 \mathrm{~A}$ ). In contrast, there were marked reductions in both outcomes during months 3-6 (figure 1B). The increase in flares in the first 3 months was most evident $(p=0.0006)$ and the decrease during the second 3 months was least marked $(\mathrm{p}=0.0006)$ in subjects receiving $\mathrm{q} 4$ pegloticase. Previous analysis indicated that q2 pegloticase caused persistently lowered plasma urate in responders, whereas $q 4$ administration resulted in a more saw tooth pattern. ${ }^{5}$ Multivariate linear regression analysis indicated that the only variable associated with flares in the treated subjects was the absolute change in plasma urate prior to these events. Assessment of plasma urate at both 2 weeks and immediately before flares indicated that larger differences between these two values were associated with increased flare occurrence $(p=0.002)$.
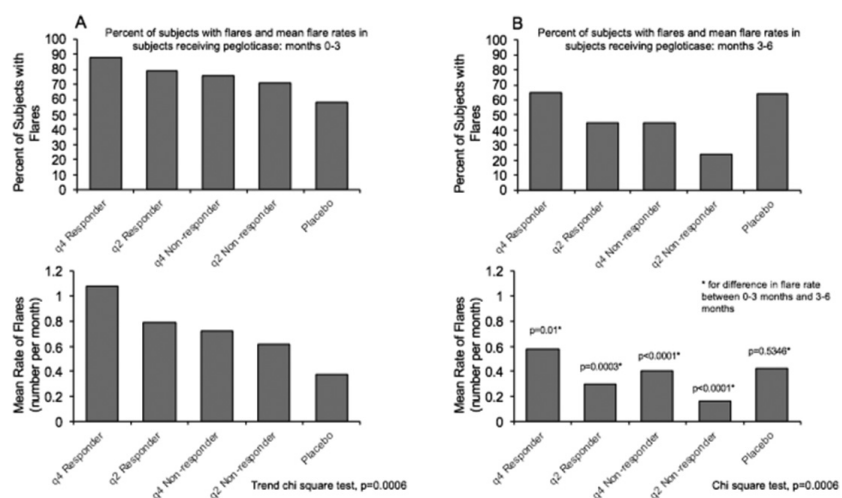

Conclusions: Flares associated with pegloticase treatment in subjects with chronic refractory gout were increased most markedly in responders to monthly administration and were significantly associated with fluctuations in plasma urate levels. Such fluctuations are much more common with monthly administration of pegloticase vs the recommended biweekly treatment regimen.

\section{REFERENCES}

[1] Wortmann RL, et al. Clin Ther 2010;32:2386.

[2] Sundy JS, et al. JAMA 2011;306:711.

[3] Mandell BF, et al. Arthritis Rheumatol 2016;68(suppl 10):212.

[4] Mandell BF, et al. Arthritis Rheumatol 2017; 69(suppl 10):2054

[5] Lipsky PE, et al. Arthritis Res Ther 2014;16:R60.

Disclosure of Interest: L. Calabrese: None declared, T. Fields Consultant for: Horizon Pharma, Takeda, Ironwood, Speakers bureau: Horizon Pharma, Takeda Ironwood, A. Yeo Consultant for: Horizon Pharma, P. Lipsky Consultant for: Horizon Pharma

DOI: 10.1136/annrheumdis-2018-eular.5961

\section{OP0185 EMERGENCY DEPARTMENT VISITS FOR GOUT: A DRAMATIC INCREASE IN THE PAST DECADE}

\section{A. Mithal ${ }^{1}$, G. Singh ${ }^{2} .{ }^{1}$ ICORE; ${ }^{2}$ Stanford University, Woodside, USA}

Background: Several studies have suggested that the prevalence of gout has been increasing worldwide, perhaps related to lifestyle factors. ${ }^{1-2}$ In the US, the NHANES study found a significantly higher age-adjusted prevalence $(3.9 \%)$ in 2007-2008 than the estimate in 1988-1994 (2.9\%). This trend paralleled an observed increase in hyperuricemia.

Objectives: To study emergency department visits due to gout in the US over 9 years (2006 to 2014)

Methods: The Nationwide Emergency Department Sample (NEDS) is the largest all-payer emergency department (ED) database in the United States, yielding national estimates of hospital-based ED visits. It contains information from 31 million ED visits at 945 hospitals in 34 states that approximate a 20-percent stratified sample of U.S. hospital-owned EDs. Weighted, it estimates roughly 143 million ED visits. We studied all ED with primary diagnosis of Gout (ICD9 code 274.xx) from 2006-2014 and calculated prevalence in different age groups. Population data was obtained from US census bureau.

Results: Over last 9 years, 1.7 million people visited EDs with a primary diagnosis of acute gout. The total number of ED visits per year increased from 168580 in 2006 to 213780 in 2014, an increase of $26.8 \%$. The prevalence of emergency room visits with primary diagnosis of gout increased from $56.5 / 100,000$ population 
in 2006 to $67 / 100,000$ in 2014 ( $p<0.001)$. The largest increase in ED visits was a $28 \%$ increase in prevalence among the 45-64 years age group from $91 / 100,000$ to $116 / 100,000(27 \%, p<0.001)$. Men were responsible for $78 \%$ of the ED visits in both 2006 and 2014.

\begin{tabular}{lcccc}
\hline $\begin{array}{l}\text { Age in } \\
\text { years }\end{array}$ & $\begin{array}{c}\text { Total number } \\
\text { of ED visits in } \\
2006\end{array}$ & $\begin{array}{c}\text { Prevalence of ED } \\
\text { visits/100,000 } \\
\text { population in 2006 }\end{array}$ & $\begin{array}{c}\text { Total number } \\
\text { of ED visits in } \\
2014\end{array}$ & $\begin{array}{c}\text { Prevalence of ED } \\
\text { visits/100,000 } \\
\text { population in 2014 }\end{array}$ \\
\hline $18-44$ & 49265 & 44 & 55216 & 48 \\
$45-64$ & 68544 & 91 & 97081 & 116 \\
$65-84$ & 44358 & 137 & 53206 & 133 \\
$85+$ & 6242 & 128 & 8126 & 132 \\
All & 205905 & 56.5 & 213780 & 67 \\
ages & & & & \\
\hline
\end{tabular}

Conclusions: Emergency Department visits have increased dramatically in the US over the last 9 years, and this increase is mostly in the 45-64 years age group perhaps reflecting the undermanaged burden of uncontrolled gout. Education, improved recognition and long-term management as well as increased use of preventive strategies is needed.

\section{REFERENCES:}

[1] Lawrence RC, et al. Estimates of the prevalence of arthritis and other rheumatic conditions in the United States. Part II. Arthritis Rheum 2008;58 (1):26-35.

[2] Kuo CF, et al. Global epidemiology of gout: Prevalence, incidence and risk factors. Nat Rev Rheumatol 2015;11(11):649-62.

Disclosure of Interest: A. Mithal: None declared, G. Singh Grant/research support from: Horizon Pharma

DOI: 10.1136/annrheumdis-2018-eular.6220

\section{OP0186 NEPHROLITHIASIS AS A COMPLICATION OF GOUT: A CROSS-SECTIONAL STUDY WITH HELICAL COMPUTED TOMOGRAPHY}

F.P. Ramos, A.T. Barbosa, R.B. Souza, F. Seragioli, R.D.N. Giorgi, R.F. Rosa. Rheumatology, IAMSPE, São Paulo, Brazil

Background: Gout is the most prevalent inflammatory arthritis, secondary to persistent hyperuricemia. It has been associated with development and progression of cardiometabolic diseases and chronic kidney disease. Several studies have shown a relationship between hyperuricemia and history of nephrolithiasis, although cut-off levels for uric acid and other related risk factors are still not well established.

Objectives: To determine the frequency of nephrolithiasis (NL) detected by helical computed tomography (h-CT) and its associated risk factors in patients with gout in a tertiary hospital of São Paulo, Brazil.

Methods: This cross-sectional study, conducted from 2016 to 2017, included 80 patients with a diagnosis of gout, according to the criteria of the ACR/EULAR2015. They were questioned about the previous history of NL and submitted to $\mathrm{h}$ $\mathrm{CT}$ for NL. Two groups were established: with and without NL; and later, unilateral and bilateral lithiasis. Anthropometric data, disease duration, serum uric acid (UA), creatinine, urinary $\mathrm{pH}(\mathrm{pH})$ and urinary $\mathrm{UA}$ of groups were compared. Statistical analysis included: mean, standard deviation, relative percentages, t-student test, chi-square test and ROC curve. Comparison of time to event-rate was performed by Kaplan-Meier method with log rank test. $p<0.05$ was considered statistically significant.

Results: NL was confirmed by h-CT in $30 \%$ of patients. However, only $16 \%$ reported previous history of NL. Groups with NL and without NL were similar in mean age $(65,96 \pm 5,54$ and $68,89 \pm 9,58$ years, $p=0.147)$, disease duration $(16,63$ $\pm 11,49$ and $11,77 \pm 9,74$ years, $p=0.056)$ and BMI $(29.72 \pm 5.09$ and $28.82 \pm 5,08$, $\mathrm{p}=0.470$ ). The $\mathrm{NL}$ group had higher pre-treatment UA compared with patients without NL $(9,36 \pm 1,09$ and $8,80 \pm 1,08 \mathrm{mg} / \mathrm{dL}, \mathrm{p}<0.05)$ and the most acidic $\mathrm{pH}$ $(5,26 \pm 0,42$ and $5.74 \pm 0.62, p<0.05)$. In addition, patients with bilateral NL presented higher BMI than unilateral patients $(p=0.036)$. According to ROC curve analysis, the best cutoff value for pre-treatment UA was $8.5 \mathrm{mg} / \mathrm{dL}$, yielding sensitivity and specificity of $75 \%$ and $50 \%$, respectively, for predicting NL events in this study. Kaplan-Meyer analysis showed that after 20 years of disease, $55 \%$ of patients with pre-treatment $\mathrm{AU} \geq 8.5 \mathrm{mg} / \mathrm{dL}$ had $\mathrm{NL}$, while only $18 \%$ of patients with pre-treatment $\mathrm{AU}<8.5 \mathrm{mg} / \mathrm{dL}$.

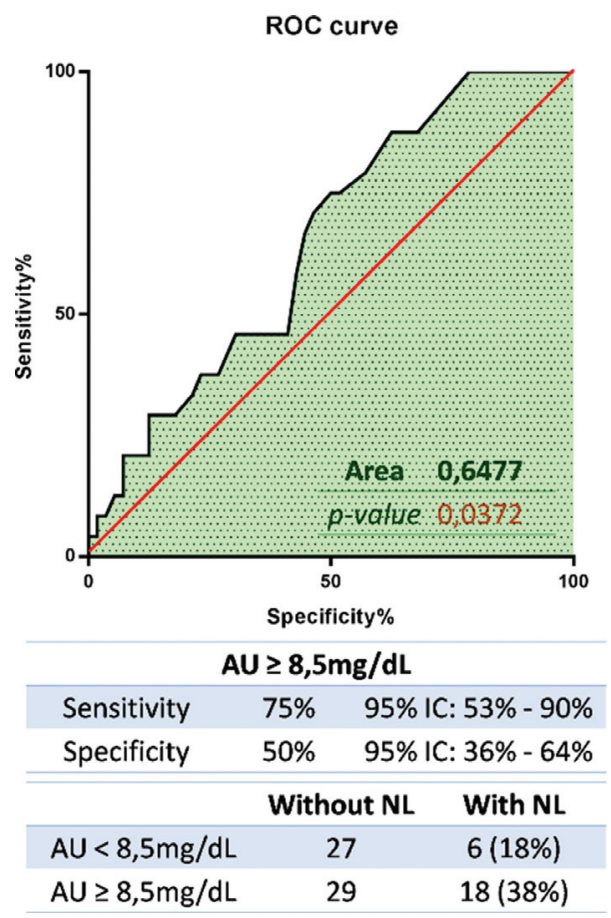

Conclusions: Since prevalence of NL in gout patients cannot be determined reliably from the clinical history, an active screening test for NL should be performed in these patients. Our study suggests that urine acidification and UA $\geq 8.5 \mathrm{mg} / \mathrm{dL}$ are associated with an increased risk of NL during follow-up of gout patients and should be corrected in their treatment.

\section{REFERENCES}

[1] Shimizu T, et al. The prevalence of nephrolithiasis in patients with primary gout: a cross-sectional study using helical computed tomography. J Rheumatol 2009 Sep;36(9):1958-62. doi:10.3899/jrheum.081128 (Epub: 2009 Jul 15).

[2] Wan KS, et al. Nephrolithiasis among male patients with newly diagnosed gout. Hong Kong Med J 2016 Dec;22(6):534-7. (Epub: 2016 Sep 9).

[3] Shimizu T, et al. Novel evaluation of nephrolithiasis as a complication of gout: a cross-sectional study using helical computerized tomography. J Urol 2013 May;189(5):1747-52. doi:10.1016/j.juro.2012.11.076 (Epub: 2012 Nov 15).

[4] Kramer $\mathrm{HJ}$, et al. The association between gout and nephrolithiasis in men: The health professionals follow-up study. Kidney Int 2003 Sep;64 (3):1022-6

Disclosure of Interest: None declared

DOI: 10.1136/annrheumdis-2018-eular.1555

\section{OP0187 FRUCTOSE-CONTAINING BEVERAGES IS AN INDEPENDENT RISK FACTOR FOR GOUT EARLY- ONSET IN SOUTH CHINA}

Q. Li' ${ }^{1}$, S.-H. Zeng ${ }^{2}$, X.-Y. Du ${ }^{1}$, Y.-H. Xu', K.-M. Yang ${ }^{1}$, J.-J. Liang ${ }^{1}$, Y.-Q. Mo ${ }^{1}$, L. Dai ${ }^{1 *}{ }^{1}$ Department of Rheumatology, Sun Yat-Sen Memorial Hospital, Sun YatSen University, ${ }^{2}$ Zhongshan School of Medicine, Sun Yat-Sen University, Guangzhou, China

Background: A trend of earlier onset of gout has been reported even though its incidence increases in a linear fashion with age until 70 years. Dietary factors have been supposed to be contributed to the early onset of gout.

Objectives: To investigate diet characteristics of gout and their impact on the early onset of gout.

Methods: Consecutive gout patients who fulfilled the 2016 ACR/EULAR classification criteria were recruited between Dec 2016 and Dec 2017 A cross-section survey on dietary factors before gout onset was conducted with semi-quantitative diet questionnaire. The questionnaire included alcohol, fructose-containing beverages, soup, animal organs, sea-foods, hotpot, tea and coffee, which impact on 